\title{
The PsbP and PsbQ family proteins in the photosynthetic machinery of chloroplasts
}

\author{
Kentaro Ifuku ${ }^{1,2, *}$ \\ ${ }^{1}$ Graduate School of Biostudies, Kyoto University, Sakyo-ku, Kyoto 606-8502, Japan \\ ${ }^{2}$ Japan Science and Technology Agency, PRESTO, 4-1-8 Honcho Kawaguchi, Saitama \\ 332-0012, Japan
}

*Corresponding author at: Graduate School of Biostudies, Kyoto University, Sakyo-ku, Kyoto 606-8502 Japan. Tel.: +81-75-753-6381, Fax: +81-75-753-6398.

E-mail address: ifuku@kais.kyoto-u.ac.jp 


\section{Abstract}

The PsbP and PsbQ proteins are extrinsic subunits of the photosystem II in eukaryotic photosynthetic organisms including higher plants, green algae and euglena. It has been suggested that PsbP and PsbQ have evolved from their cyanobacterial homologs, while considerable genetic and functional modifications have occurred to generate the eukaryote-type proteins. In addition, number of PsbP and PsbQ homologs exist in the thylakoid lumen of chloroplasts. These homologs are nuclear-encoded and likely diverged by gene duplication, and recent studies have elucidated their various functions in the photosynthetic machinery. In this short review, recent findings and new idea about these components will be discussed.

Keywords: extrinsic proteins; molecular evolution; oxygen-evolving complex; photosystem II; PsbP; PsbQ

Abbreviation: Cyt, cytochrome; Fd, ferredoxin; FTIR, Fourier transform infrared; LHCII, light-harvesting complex II; NDH, NAD(P)H dehydrogenase; OEC, oxygen-evolving complex; PSII, photosystem II; PPD, PsbP-domain; PPL, PsbP-like; PQL, PsbQ-like 


\section{Introduction}

In eukaryotic photosynthetic organisms, oxygenic photosynthesis takes place in chloroplasts, in which protein complexes involved in the light-harvesting and photosynthetic electron transport are located in thylakoid membranes. Photosynthetic electron transport machinery is composed of two light-energy driven photosystems (PS), PSI and PSII, the cytochrome (Cyt) $b_{6} f$, and the ATP synthase. In linear electron transport (LET), electrons extracted from water in PSII are transported to PSI through Cyt $b_{6} f$ and eventually produce NADPH. During this process, PSII oxidizes water thereby producing molecular oxygen and protons in the thylakoid luminal space. In addition, protons are concomitantly transferred from the stroma to the lumen and the resulting $\Delta \mathrm{pH}$ is used to drive ATP production in the ATP synthase. Furthermore, $\Delta \mathrm{pH}$ is additionally formed by cyclic electron transport around PSI (PSI-CEF), which recycles electrons from ferredoxin (Fd) or NADPH to the plastoquinone pool. In PSI-CEF, the existence of two different electron transport pathways has been demonstrated; One is an antimycin-sensitive complex including PGR5 and PGRL1 proteins (Hertle et al. 2013, Leister and Shikanai 2013) and the other is the NAD(P)H dehydrogenase-like (NDH-like) complex (Peng et al. 2009, 2011; Ifuku et al. 2011a), both of which are suggested to interacts with PSI.

The biogenesis and regulation of the multi-subunits complexes in thylakoid membranes have been intensively studied, and recent studies suggest the importance of the proteins accumulated in thylakoid luminal space. Proteomic and genomic studies have identified up to 80 proteins in Arabidopsis to be localized in the lumen (Peltier et al. 2002; Schubert et al. 2002; Kieselbach and Schroder, 2003). Since recent review article has already described known and proposed functions of thylakoid luminal proteins in photosynthesis regulation (Järvi et al. 2013), only a brief summary on the functional category of luminal proteins is shown in Fig. 1. In addition to the major luminal proteins, such as PSI and PSII extrinsic subunits, the electron carrier plastcyanin (PC), and violaxanthin deepoxigenase (VDE), a number of proteins involved in protein folding and quality control, such as immunophilins (CYP- and FKBP-types) and Deg proteases have been identified (Schuhmann and Adamska, 2012; Gollan et al. 2012). Furthermore, several enzymes involved in the thiol/disulphide modulation has been identified, suggesting the importance of redox regulation and sensing in thylakoid lumen (Hall et al. 2010; Järvi et al. 2013); Lumen Thiol Oxidoreductase1 (LTO1) catalyzes disulphide bond formation of the PsbO subunit in PSII (Karamoko et al. 2011), and CS26 was proposed to regulate thiol 
oxidation by production of S-sulphocysteine in the lumen (Bermúdez et al., 2012). These facts indicate the protein interactive network in thylakoid lumen that supports functions of the photosynthetic electron transfer machinery.

In this review, I would like to focus on the PsbP and PsbQ family, one of the major protein families in the thylakoid lumen. The PsbP and PsbQ proteins are extrinsic subunits of the photosystem II in green plants including higher plants and green algae (Enami et al. 2008). It is known that the PSII extrinsic proteins have undergone quite drastic changes during the evolution of oxyphototrophs from cyanobacteria to higher plants (De Las Rivas et al. 2004; Roose et al. 2007; Enami et al. 2008). The origins of PsbP and PsbQ are thought to be their cyanobacterial homologs CyanoP and CyanoQ, respectively (Kashino et al. 2002, Thornton et al. 2004); however, it has been suggested that the molecular functions of CyanoP and CyanoQ in the oxygen-evolving complex (OEC) are largely different from PsbP and PsbQ. In red algae and diatoms, PsbQ', a $20 \mathrm{kDa}$ homologue of CyanoQ, is bound to PSII as an extrinsic subunits (Okumura et al. 2008, Nagao et al. 2010b). In addition, diatoms have specific extrinsic subunit, Psb31, and recent structural analysis suggests that Psb31 might be a homolog of PsbQ (Nagao et al. 2013). Furthermore, genomic and proteomic studies have identified a number of PsbP and PsbQ homologs in chloroplasts. All PsbP and PsbQ homologs are nuclear-encoded and likely result from gene duplication (Roose et al. 2007). This review summarizes recent findings and new idea about their function and molecular evolution in the photosynthetic electron machinery.

\section{Members of the PsbP and PsbQ family in Arabidopsis}

\subsection{Members of the PsbP family}

The structure and function of the PsbP family proteins have recently been thoroughly reviewed (Bricker et al. 2013), and only the essence will be descried here. In Arabidopsis, at least nine members of PsbP family proteins, PsbP and eight PsbP homologs, are accumulated in thylakoid lumen (Table 1) (Roose et al. 2007; Ishihara et al. 2007; Ifuku et al. 2010). Based on the amino-acid sequence similarity with PsbP, PsbP homologs are classified into two PsbP-like proteins (PPL) and six PsbP-domain proteins (PPD) (Ishihara et al. 2007). The presence of additional members of the PsbP family, which are named as PPD7 or PPD8 have been suggested, but they have not been detected by proteome studies (Sato, 2010; Järvi et al. 2013).

Comparison of amino acid sequences suggests that PPL1 is most closely related to CyanoP, 
while PsbP and the other PsbP homologs are paralogs of PPL1 (Ishihara et al. 2007; Sato 2010; Jackson et al. 2012). Structural modeling in silico (Sato, 2010) suggests that all members of the PsbP family have a similar $\alpha \beta \alpha$ structure, called a Mog1p/PsbP-like fold in SCOP: a structural classification of proteins database (Ifuku et al. 2004). On the other hand, the N- or C- terminal sequence as well as the loop region connected to the central $\beta$-sheets differ among the members (Sato 2010). It has been suggested that this structural difference among PsbP family proteins should be related to their functional difference in the thylakoid lumen (Sato 2010; Bricker et al. 2013). In particular, the N-terminal extension specifically found in PsbP is required for its stable interaction with PSII and for its function to retain essential $\mathrm{Ca}^{2+}$ and $\mathrm{Cl}^{-}$ions in PSII (Ifuku et al. 2002, 2008; Tomita et al. 2009).

\subsection{Members of the PsbQ family}

In addition to two authentic PsbQs in PSII, Arabidopsis has three PsbQ-like (PQL) homologs (Table 1). All five PsbQ family proteins have an obvious thylakoid lumen-targeting signal, consistent with previous proteomic studies which suggested their localization in the thylakoid lumen, except for PQL3 (Peltier et al. 2002; Schubert et al. 2002; Zybailov et al. 2008). It should be noted that three PQL proteins were given different names by different groups (Peng et al. 2009; Suorsa et al. 2010; Yabuta et al. 2010), and unified names have been proposed (Ifuku et al. 2011a), which will be described in the following section. Comparison of amino-acid sequences suggests that the PQL proteins are more similar to plant PsbQ than CyanoQ, indicating the possibility that PQLs may have been differentiated from PsbQ after PsbQ had evolved from CyanoQ (Yabuta et al. 2010).

A phylogenetic analysis has investigated the evolutional relationship among PsbQ family proteins (Yabuta et al. 2010). Structural modeling indicates that all of the PQL proteins are predicted to have the four-helix bundle structure similar to PsbQ and CyanoQ (Calderon et al. 2003; Balsera et al. 2005), and only this sequence region was used to construct a reliable tree. The obtained tree suggests that PsbQ and PQL branched after PsbQ and PsbQ' had branched from CyanoP. It should be noted that PsbQ in green algae is more closely related to PsbQ' in red algae and diatoms, indicating that PsbQ in green algae may be included in the PsbQ' group. This assumption is consistent with the different binding properties of green algal PsbQ from higher plant PsbQ in the PSII complex (Nagao et al. 2010a). Recently, structural analysis of Psb31 from a centric diatom, Chaetoceros glacilis, suggests that Psb31 has a four-helix bundle structure similar to the PsbQ family protein (Nagao et al. 2013); However, Psb31 proteins in 
diatom are significantly distant from other PsbQ family proteins. Because the four-helix bundle structure is commonly observed in other PSII interacting proteins, such as Psb27 (Cormann et al. 2009; Mabbitt et al. 2009; Michoux et al. 2012) and CYP38 (Vasudevan et al. 2012), it is difficult to conclude from the structure the evolutional origin of Psb31 as CyanoQ. The above phylogenetic analysis suggests that the PsbQ and PQL proteins should be evolutionally new proteins with specific roles in land plants.

\subsection{Functional prediction of the PsbP and $P s b Q$ family using $m R N A$ co-expression analysis}

Molecular functions of the PsbP and PsbQ family proteins have been investigated by the mRNA co-expression patterns in the public available microarray database (Ishihara et al 2007; Yabuta et al. 2010). Comprehensive analyses on the mRNA co-expression of thylakoid luminal protein genes suggest physiologically sensible sub-grouping among the luminal protein genes (Granlund et al. 2009; Ifuku et al. 2010). It is suggested that PsbP and PsbQ homologs in Arabidopsis are categorized into three distinct groups; The first group contains the authentic OEC proteins of PSII, which mainly have PSI or PSII subunits in their co-expression genes. The second group co-expresses with ribosomes, immunophilines and some stress-related genes, and the third group characteristically co-expresses with the genes encoding subunits of chloroplast NDH-like complex (Ifuku et al. 2010). This functional classification corresponds well with the actual phenotype of the Arabidopsis mutants lacking PPL and PQL proteins. On the other hand, recent studies suggest that this classification based on the mRNA co-expression is not necessarily true for the other PPD proteins (Roose et al. 2011, Liu et al. 2012).

\section{Functions of PsbP and PsbQ family proteins in photosystem II}

\subsection{Functions of PsbP and PsbQ proteins in the OEC}

Numerous studies have characterized the function of PsbP and PsbQ proteins at the OEC in PSII to optimize the availability of $\mathrm{Ca}^{2+}$ and $\mathrm{Cl}^{-}$cofactors to maintain the active $\mathrm{Mn}$ cluster in PSII, which have been intensively reviewed (Seidler et al. 1996; Bricker et al. 2012; Ifuku et al. 2008, 2011). Nevertheless, it has been still elusive how PsbP and PsbQ function to stabilize the binding of the inorganic cofactors and enhance oxygen-evolution in PSII. To answer this question, Noguchi and coworkers applied the Fourier transform infrared (FTIR) measurement and succeeded to detect the conformational changes of the OEC induced by PsbP binding upon 
the $S_{1}$ to $S_{2}$ transition of the Mn Cluster (Tomita et al. 2009).

Figure 2 shows the summary of the results obtained from the FTIR analyses. They demonstrate that the $\mathrm{N}$-terminal sequence of PsbP, which is essential to retain $\mathrm{Ca}^{2+}$ and $\mathrm{Cl}^{-}$in PSII, is indispensable to induce the proper conformational changes around the OEC (Tomita et al. 2009). Because neither PsbQ nor PsbO is responsible for this conformational change, PsbP binding directly affects the conformation in the intrinsic proteins. Consistently, a recent study using a chemical crosslinker EDC demonstrates that Ala1 in the PsbP N-terminus has a charge-pair interaction with Glu57 in the membrane- intrinsic PsbE (Cyt $b_{559} \alpha$ subunit) (Ido et al. 2012). This is the first direct evidence showing the interaction between PsbP and the PSII intrinsic subunit in higher plants system, while an interaction between PsbP and PsbE was suggested in green algal PSII (Nagao et al. 2010b). It was pointed out that in the cyanobacterial PSII structure, the N-terminus of PsbV (Ala1) appears to interact with Asp53 of PsbE (Bricker et al. 2013). It is therefore possible that in higher plants PsbP may occupy a position similar to PsbV and induce conformational changes to retain $\mathrm{Ca}^{2+}$ and $\mathrm{Cl}^{-}$in the OEC of PSII that might be induced by PsbV in cyanobacterial PSII. Further FTIR study using cyanobacterial system will address this point.

It has been proposed that PsbQ supports the PsbP function (Ifuku and Sato 2002). In fact, a recent FTIR study demonstrates that PsbQ restored the ability of the N-terminal truncated PsbP to induce the proper conformational changes upon the $S_{1}$ to $S_{2}$ transition (Kakiuchi et al. 2012) (Fig. 2f). This observation is interesting because in Synechocystis sp. PCC 6803, the major function of CyanoQ is to stabilize the PsbV binding to PSII, thereby contributing to the protection of the catalytic Mn cluster of the OEC (Kashino et al. 2006). In red algal PSII, PsbQ' is required for the stable binding of PsbV (Enami et al. 1998), and recent FTIR studies suggest that the function of PsbV is to retain a proper OEC conformation, which corresponds to that of PsbP in higher plants (Uno et al. 2013). Direct interaction between PsbP and PsbQ is indicated in Chlamydomonas PSII (Nagao et al. 2010). Presumably, molecular function of CyanoQ, PsbQ', and PsbQ is partly conserved during evolution, while the interacting partner has been changed from PsbV to PsbP in green plants.

\subsection{Function of PsbP and $P s b Q$ proteins in the dynamic PSII function}

PSII is not a static structure and it undergoes constant assembly, degradation and repair (Nickelsen and Rengstl, 2013). Furthermore, the association of peripheral antennae to PSII is a dynamic process that facilitates adjustment of photosynthetic light reactions to environmental 
changes. Recent studies suggest that PsbP and PsbQ play an important role in these dynamic processes. It has been suggested that PsbP proteins would function as an assembly and/or stability factor for PSII in Chlamydomonas and higher plants (Bricker et al. 2013). This view is supported by a recent study showing that complete elimination of PsbP in an Arabidopsis mutant impairs photoautotrophy causing a seedling-lethal phenotype (Allahverdiyeva et al. 2013). On the other hand, non-complete suppression of PsbP by RNAi (5-10\% of the wild type levels) allows slow photoautotrophic growth and accumulation of the PSII reaction center close to the normal level, whereas functional defects in both the oxidizing and reducing side of PSII was observed (Ifuku et al. 2005; Yi et al. 2007; Ido et al. 2009). These facts suggest that PsbP would have dual functions in PSII: One is the "catalytic" function like a molecular chaperon with respect to the PSII assembly (Bricker et al 2012), and the other is structural roles as the OEC subunits that protect the Mn cluster in PSII (Ishihara et al. 2005). PsbQ may not be required for the former process, but should be involved in the latter by stabilizing the binding of PsbP.

As already mentioned above, PsbP and PsbQ are also suggested to play an important role in defining the architecture of PSII-light-harvesting complex (LHC) II supercomplexes in higher plants (Caffarri et al., 2009; Ifuku et al., 2011b). PsbP knockdown by RNAi in tobacco resulted in a severe decrease in the amount of PSII-LHCII super-complexes, while amounts of unattached LHCII trimers and minor LHCs were significantly increased (Ido et al., 2009). Similarly, the abundance of PSII-LHCII supercomplexes decreased in the mutants lacking PsbQ and/or PsbR (Allahverdiyeva et al. 2013). Since both PsbQ and PsbR have a function to stabilize the binding of PsbP to PSII, these three components have specific and important roles in stabilizing the PSII-LHCII super-complexes. Furthermore, depletion of PsbQ and/or PsbR has a major effect on short-term regulatory mechanisms, such as state transitions and non-photochemical quenching (Allahverdiyeva et al. 2013). The absence of those subunits in the mutants accelerates the rate of the state changes, which would be caused by the increase of the LHCII antenna loosely bound to PSII.

It has been reported that PsbP, PsbQ, and PsbR can be phosphorylated in the thylakoid lumen (Reiland et al. 2009). It is therefore possible that phosphorylation of these luminal components affects the assembly of PSII, although any luminal kinases has not been identified yet. Recent study suggests that TLP18.3 protein in thylakoid lumen is a novel acid phosphatase (Wu et al., 2011) and important for the PSII assembly (Sirpio et al., 2007), while its substrates have not been identified. Further studies are obviously required to understand the physiological 
significance of this phosphorylation event in the thylakoid lumen.

\subsection{Function of other PsbP homologs in the PSII complex}

A previous study suggests that PsbP-like protein 1 (PPL1) is required for the efficient repair of photo-damaged PSII (Ishihara et al. 2007). In mRNA co-expression analysis using the ATTED-II database (Obayashi et al. 2007, 2009), PPL1 is included in stress-related groups and co-expresses with luminal immunophilines such as CYP38 (Sirpio et al. 2008), FKBP13 (Gupta et al. 2002; Gopalan et al. 2004), which are involved in the assembly of PSII and Cyt $b_{6} f$ complex, respectively. Characterization using the PPL1-RNAi plants shows that the extent of photo-sensitivity is correlated with the levels of PPL accumulation (Matsui et al. 2013). It is also mentioned that PPL1 is mainly localized in the stroma-exposed (unstacked) thylakoid regions where the biogenesis and repair of PSII take place. These facts support the idea that PPL1 would have a function to support the formation or assembly of the PSII complex; however, association of PPL1 with PSII has not been detected experimentally. The PPD3 and PPD6 proteins were also hypothesized to have a function related to PSII, because several PSII subunits are included in the co-expression network (Ifuku et al. 2010). Further studies will elucidate the function of those PsbP homologs in PSII.

\section{Functions of the PsbP and PsbQ family proteins in the chloroplast NDH-like complex}

\subsection{Structure of the chloroplast NDH-like complex}

The chloroplast NDH-like complex is assembled from five distinct subcomplexes in vascular plants (Fig. 3) (Suorsa et al., 2009; Ifuku et al., 2011a; Peng et al., 2011a; Yamamoto et al., 2011). It consists of the membrane subcomplex, the stroma-exposed hydrophilic subcomplex A, the subcomplex B that attached to the membrane subcomplex, the subcomplex on the luminal side, and the electron donor binding subcomplex that accepts $\mathrm{Fd}$ as an electron donor. The membrane subcomplex and the subcomplex A are conserved in cyanobacteria, while the subunits in the subcomplex B, 'lumen', and 'donor-binding' are all encoded by nuclear genes and specific to angiosperms (Peng et al., 2009; Yamamoto et al., 2011). Moreover, chloroplast NDH interacts with PSI to form the NDH-PSI supercomplex in Arabidopsis, which is required for the stabilization of the NDH-like complex (Peng et al., 2008, 2009; Peng and Shikanai, 2011). Two minor light-harvesting complex I proteins, Lhca5 and Lhca6, function as a 
linker required for the interaction between NDH and PSI (Peng et al., 2009). Consequently, the chloroplast NDH-like complex is among the largest protein complexes in thylakoid membranes.

As indicated by the mRNA coexpression analysis, several PsbP and PsbQ family proteins are included in the NDH-like complex; the PPL2 and two PQL proteins have been shown as the subunits of the luminal subcomplex (Ishihara et al. 2007; Peng et al. 2009; Suorsa et al. 2010; Yabuta et al. 2010). Two PQLs were given different names by different groups (Peng et al. 2009, Suorsa et al. 2010, Yabuta et al. 2010). To avoid confusion, PPL2 is renamed as PnsL1, and two PQLs as PnsL2 (At1g14150) and as PnsL3 (At1g14150), where "PnsL" represents Photosynthetic NDH subcomplex Lumen (Ifuku et al. 2011). Although PQL3 (At2g01918) is also required for the stable accumulation and activity of NDH (Yabuta et al. 2010), the nomenclature of PQL3 is not changed since its cellular localization is not clear. Interestingly, two types of immunophilin, FKBP16-2 and CYP20-2, are also included in the luminal subcomplex in Arabidopsis (Peng et al. 2009, Sirpiö et al. 2009b). Biochemical analyses suggest that PnsL1 (PPL2), PnsL2 (At1g14150), PnsL4 (FKBP16-2), and PnsL5 (CYP20-2) form luminal subcomplex that are connected to stromal subcomplex A, while PsnL3 (At1g14150) shows a different location and is associated with subcomplex B (Suorsa et al. 2010; Yabuta et al. 2010).

\subsection{Functions of PsbP-and PsbQ like proteins in the chloroplast $N D H$-like complex}

The subunits of the luminal subcomplex in the chloroplast NDH-like complex does not exist in the liverwort Marchantia polymorpha and the moss Physcomitrella patens, implying that the luminal supercomplex including PPL and PQLs represents a relatively recent evolutionary acquisition. In addition, the NDH-PSI supercomplex is not detected in $M$. polymorpha that lacks the linker protein Lhca5 and Lhca6 (Ueda et al., 2012), and only small amount is found in P. patens that has only Lhca5 (Ambruster et al. 2013). These facts suggest that the PsbP and PsbQ homologs together with several immunophilines and Lhc proteins have evolved to facilitate and/or stabilize the highly-ordered structure of the NDH-PSI supercomplex, as observed in the PSII-LHCII supercomplex.

Very interestingly, a recent study indicates that PAM68L, a homolog of PAM68, a photosystem II assembly factor, mediates the assembly of the chloroplast NDH-like complex in Arabidopsis (Ambruster et al. 2013). PAM68L does not exsit in M. polymorpha and P. patens and is suggested to facilitate the incorporation of the luminal subcomplex into other NDH-like subcomplexes. Thus, the NDH-like complex of vascular plants recruited subunits related to 
function in the PSII complex and utilizes them as both structural components and assembly factors. Presumably, the PsbP and PsbQ homologs might be originally assembly factors and have been developed as indispensable subunits for the NDH-like complex during evolution (Suorsa et al. 2009). It is also possible that luminal subunits may regulate the oligomeric state and stability of the NDH-PSI complex.

\section{Other functions of the PsbP-domain proteins}

Although the mRNA coexpression analysis did not suggest the specific function of PPD1, it is recently reported that PPD1 is essential for the assembly of PSI (Liu et al. 2012). Deletion of PPD1 results in an inability of the mutant to grow photoautotrophically and a specific loss of the stable PSI complex. Biochemical and molecular biological analyses reveal that PPD1 interacts directly and specifically with PsaA and PsaB and assists their proper folding and integration into thylakoid membranes. PPD1 homolgs have not been identified in cyanobacteria (Ifuku et al. 2010), indicating the specific role in eukaryotic photosynthetic organisms.

Molecular function of other PPD proteins has also been investigated. PPD2 protein is proposed to be involved in singlet-oxygen signaling in Chlamydomonas (Brzezowski et al. 2012), while PPD5 has been linked to plant development via the strigolactone synthesis (Roose et al. 2011). Although those observations are very intriguing, their function in the photosynthetic machinery is still not clear and will not be discussed here. The current status of the knowledge about these PPD proteins is reviewed by Bricker et al. (2013).

\section{Conclusion}

As described in this review, the PsbP and PsbQ family proteins function not only as structural components but also as assembly factors of various photosynthetic machineries in chloroplasts. In particular, the PsbP protein in PSII appears to preserve both functions and has essential roles to maintain the photosynthetic oxygen evolution in PSII (Ifuku et al. 2005; Yi et al. 2007). It is probable that the PsbP and PsbQ homologs may cooperate with the thylakoid luminal immunophilins to assist the correct folding and functional assembly of the thylakoid membrane protein complexes, such as the PSII and NDH-like complexes. It is tempting to speculate that these luminal subunits also have regulatory roles in the photosynthetic electron transport under changing environments. Recent finding suggesting the redox regulation and phosphorylation of thylakoid luminal proteins support this view. Further genetic, molecular biological, and biochemical analyses are required to elucidate this issue. 


\section{Acknowledgments}

This work was supported in part by a grant from JST PRESTO (to Ke.I.) and by Grant-in-Aid for Young Scientists (B) from JSPS (Grant no. 18770032 to Ke.I.). 


\section{References}

Allahverdiyeva Y, Suorsa M, Rossi F, Pavesi A, Kater MM, Antonacci A, Tadini L, Pribil M, Schneider A, Wanner G, Leister D, Aro EM, Barbato R, Pesaresi P (2013) Arabidopsis plants lacking PsbQ and PsbR subunits of the oxygen-evolving complex show altered PSII super-complex organization and short-term adaptive mechanisms. Plant J. 75, 671-684.

Armbruster U, Rühle T, Kreller R, Strotbek C, Zühlke J, Tadini L, Blunder T, Hertle AP, Qi Y, Rengstl B, Nickelsen J, Frank W, Leister D (2013) The PHOTOSYNTHESIS AFFECTED MUTANT68-LIKE Protein Evolved from a PSII Assembly Factor to Mediate Assembly of the Chloroplast $\mathrm{NAD}(\mathrm{P}) \mathrm{H}$ Dehydrogenase Complex in Arabidopsis. Plant Cell 25, 3926-3943.

Bermúdez MÁ, Galmés J, Moreno I, Mullineaux PM, Gotor C, Romero LC (2012) Photosynthetic adaptation to length of day is dependent on S-sulfocysteine synthase activity in the thylakoid lumen. Plant Physiol. 160, 274-288.

Balsera M, Arellano JB, Revuelta JL, de las Rivas J, Hermoso JA (2005) The 1.49 A resolution crystal structure of PsbQ from photosystem II of Spinacia oleracea reveals a PPII structure in the N-terminal region. J. Mol. Biol. 350, 1051-1060.

Bricker TM, Roose JL, Fagerlund RD, Frankel LK, Eaton-Rye JJ (2012) The extrinsic proteins of Photosystem II. Biochim Biophys Acta. 1817, 121-42.

Bricker TM, Roose JL, Zhang P, Frankel LK (2013) The PsbP family of proteins. Photosynth Res. 116, 235-250.

Brzezowski P, Wilson KE, Gray GR (2012) The PSBP2 protein of Chlamydomonas reinhardtii is required for singlet oxygen-dependent signaling. Planta 236, 1289-303.

Caffarri S, Kouril R, Kereïche S, Boekema EJ, Croce R (2009) Functional architecture of higher plant photosystem II supercomplexes. EMBO J. 28, 3052-3063. 
Calderone V, Trabucco M, Vujicic A, Battistutta R, Giacometti GM, Andreucci F et al. (2003) Crystal structure of the PsbQ protein of Photosystem II from higher plants. EMBO Rep. 4, 900-905.

Cormann KU, Bangert JA, Ikeuchi M, Rögner M, Stoll R, Nowaczyk MM (2009) Structure of Psb27 in solution: implications for transient binding to photosystem II during biogenesis and repair. Biochemistry 48, 8768-8770.

De Las Rivas J, Balsera M, Barber J (2004) Evolution of oxygenic photosynthesis: genome-wide analysis of the OEC extrinsic proteins. Trends Plant Sci. 9, 18-25.

Enami I, Kikuchi S, Fukuda T, Ohta H, Shen JR (1998) Binding and functional properties of four extrinsic proteins of photosystem II from a red alga, Cyanidium caldarium, as studied by release-reconstitution experiments. Biochemistry 37, 2787-2793.

Enami I, Okumura A, Nagao R, Suzuki T, Iwai M, Shen, JR (2008) Structures and functions of the extrinsic proteins of photosystem II from different species. Photosynth. Res. 98, 349-363.

Gollan PJ, Bhave M, Aro EM (2012) The FKBP families of higher plants: Exploring the structures and functions of protein interaction specialists. FEBS Lett. 586, 3539-3547.

Granlund I, Hall M, Kieselbach T, Schröder WP (2009) Light induced changes in protein expression and uniform regulation of transcription in the thylakoid lumen of Arabidopsis thaliana. PLoS One. 4, e5649.

Gupta R, Mould RM, He Z, Luan S (2002) A chloroplast FKBP interacts with and affects the accumulation of Rieske subunit of cytochrome bf complex. Proc. Natl. Acad. Sci US A. 99, 15806-15811.

Hall M, Mata-Cabana A, Akerlund HE, Florencio FJ, Schröder WP, Lindahl M, Kieselbach T (2010) Thioredoxin targets of the plant chloroplast lumen and their implications for plastid function. Proteomics. 10, 987-1001. 
Hertle AP, Blunder T, Wunder T, Pesaresi P, Pribil M, Armbruster U, Leister D (2013) PGRL1 is the elusive ferredoxin-plastoquinone reductase in photosynthetic cyclic electron flow. Mol Cell. 49, 511-523.

Ido K, Ifuku K, Yamamoto Y, Ishihara S, Murakami A, Kusano M et al. (2009) Knockdown of the PsbP protein does not prevent assembly of the dimeric PSII core complex but impairs accumulation of photosystem II supercomplexes in tobacco. Biochem. Biophys. Acta 1787, 873-881.

Ido K, Kakiuchi S, Uno C, Nishimura T, Fukao Y, Noguchi T, Sato F, Ifuku K (2012) The conserved His-144 in the PsbP protein is important for the interaction between the PsbP N-terminus and the Cyt $b_{559}$ subunit of photosystem II. J Biol Chem. 287, 26377-26387.

Ifuku K, Endo T, Shikanai T, Aro EM (2011a) Structure of the chloroplast NADH dehydrogenase-like complex: nomenclature for nuclear-encoded subunits. Plant Cell Physiol. $52,1560-1568$.

Ifuku K, Ido K, Sato F (2011b) Molecular functions of PsbP and PsbQ proteins in the photosystem II supercomplex, J Photochem Photobiol B. 104, 158-164.

Ifuku K, Ishihara S, Shimamoto R, Ido K, Sato F (2008) Structure, function, and evolution of the PsbP protein family in higher plants. Photosynth. Res. 98, 427-437.

Ifuku K, Ishihara S, Sato F (2010) Molecular functions of oxygen-evolving complex family proteins in photosynthetic electron flow. J Integr Plant Biol. 52, 723-734.

Ifuku K, Nakatsu T, Kato H, Sato F (2004) Crystal structure of the PsbP protein of Photosystem II from Nicotiana tabacum. EMBO Rep. 5, 362-367.

Ifuku K, Sato F (2002) A truncated mutant of the extrinsic 23-kDa protein that absolutely requires the extrinsic 17-kDa protein for $\mathrm{Ca}^{2+}$ retention in photosystem II. Plant Cell Physiol. 43, 1244-1249. 
Ifuku K, Yamamoto Y, Ono TA, Ishihara S, Sato F (2005) PsbP protein, but not PsbQ protein, is essential for the regulation and stabilization of Photosystem II in higher plants. Plant Physiol. $139,1175-1184$.

Ishihara S, Takabayashi A, Ido K, Endo T, Ifuku K, Sato F (2007) Distinct functions for the two PsbP-like proteins PPL1 and PPL2 in the chloroplast thylakoid lumen of Arabidopsis. Plant Physiol. 145, 668-679.

Ishihara S, Yamamoto Y, Ifuku K, Sato F (2005) Functional analysis of four members of PsbP family in Photosystem II in Nicotiana tabacum using differential RNA interference. Plant Cell Physiol. 46, 1885-1893.

Jackson SA, Hinds MG, Eaton-Rye JJ (2012) Solution structure of CyanoP from Synechocystis sp. PCC 6803: new insights on the structural basis for functional specialization amongst PsbP family proteins. Biochim Biophys Acta. 1817, 1331-1338.

Järvi S, Gollan PJ, Aro EM (2013) Understanding the roles of the thylakoid lumen in photosynthesis regulation. Front. Physio. 4, 434.

Kakiuchi S, Uno C, Ido K, Nishimura T, Noguchi T, Ifuku K, Sato F (2012) The PsbQ protein stabilizes the functional binding of the PsbP protein to photosystem II in higher plants. Biochim Biophys Acta. 1817, 1346-1351.

Kashino Y, Inoue-Kashino N, Roose JL, Pakrasi HB, Absence of the PsbQ protein results in destabilization of the PsbV protein and decreased oxygen evolution activity in cyanobacterial photosystem II, J. Biol. Chem. 281 (2006) 20834-20841.

Kieselbach T, Schröder WP (2003) The proteome of the chloroplast lumen of higher plants. Photosynth Res. 78, 249-264.

Leister D, Shikanai T (2013) Complexities and protein complexes in the antimycin A-sensitive pathway of cyclic electron flow in plants. Front Plant Sci. 4, 161. 
Liu J, Yang H, Lu Q, Wen X, Chen F, Peng L, Zhang L, Lu C (2012) PsbP-domain protein1, a nuclear-encoded thylakoid lumenal protein, is essential for photosystem I assembly in Arabidopsis. Plant Cell 24, 4992-5006.

Mabbitt PD, Rautureau GJ, Day CL, Wilbanks SM, Eaton-Rye JJ, Hinds MG (2009) Solution structure of Psb27 from cyanobacterial photosystem II. Biochemistry 248, 8771-8773.

Matsui S, Ishihara S, Ido K, Ifuku K, and Sato, F. (2013) Functional analysis of PsbP-like protein 1 (PPL1) in Arabidopsis, In: K. Tingyun, L. Congming \& Z. Lixin (eds.) Photosynthesis Research for Food, Fuel and the Future: 15th International Congress on Photosynthesis, pp 415-417, Springer Berlin Heidelberg.

Michoux F, Takasaka K, Boehm M, Komenda J, Nixon PJ, Murray JW (2012) Crystal structure of the Psb27 assembly factor at $1.6 \AA$ A: implications for binding to Photosystem II. Photosynth Res. 110, 169-175.

Nagao R, Suga M, Niikura A, Okumura A, Koua FH, Suzuki T, Tomo T, Enami I, Shen JR (2013) Crystal Structure of Psb31, a Novel Extrinsic Protein of Photosystem II from a Marine Centric Diatom and Implications for Its Binding and Function, Biochemistry. 52, 6646-6652.

Nagao R, Moriguchi A, Tomo T, Niikura A, Nakajima S, Suzuki T, Okumura A, Iwai M, Shen JR, Ikeuchi M, Enami I (2010a) Binding and functional properties of five extrinsic proteins in oxygen-evolving photosystem II from a marine centric diatom, Chaetoceros gracilis. J. Biol. Chem. 285, 29191-29199.

Nagao R, Suzuki T, Okumura A, Niikura A, Iwai M, Dohmae N, Tomo T, Shen JR, Ikeuchi M, Enami I (2010) Topological analysis of the extrinsic PsbO, PsbP and PsbQ proteins in a green algal PSII complex by cross-linking with a water-soluble carbodiimide. Plant Cell Physiol. 51, 718-727.

Nickelsen J, Rengstl B (2013) Photosystem II assembly: from cyanobacteria to plants. Annu. Rev. Plant Biol. 64, 609-635. 
Obayashi T, Kinoshita K, Nakai K, Shibaoka M, Hayashi S, Saeki M et al. (2007) ATTED-II: a database of co-expressed genes and cis elements for identifying co-regulated gene groups in Arabidopsis. Nucleic Acids Res. 35, Database issue D863-869.

Obayashi T, Hayashi S, Saeki M, Ohta H, Kinoshita K (2009) ATTED-II provides coexpressed gene networks for Arabidopsis. Nucleic Acids Res. 37, Database issue D987-991.

Peltier JB, Emanuelsson O, Kalume DE, Ytterberg J, Friso G, Rudella A et al. (2002) Central functions of the lumenal and peripheral thylakoid proteome of Arabidopsis determined by experimentation and genome-wide prediction. Plant Cell 14, 211-236.

Peng L, Fukao Y, Fujiwara M, Takami T, Shikanai T (2009) Efficient operation of NAD(P)H dehydrogenase requires supercomplex formation with photosystem I via minor LHCI in Arabidopsis. Plant Cell 21, 3623-3640.

Peng L, Shikanai T (2011) Supercomplex formation with photosystem I is required for the stabilization of the chloroplast NADH dehydrogenase-like complex in Arabidopsis. Plant Physiol. 155, 1629-139.

Peng L, Shimizu H, Shikanai T (2008) The chloroplast NAD(P)H dehydrogenase complex interacts with photosystem I in Arabidopsis. J. Biol. Chem. 283, 34873-34879.

Peng L, Yamamoto H, Shikanai T (2011) Structure and biogenesis of the chloroplast NAD(P)H dehydrogenase complex. Biochim. Biophys. Acta. 1807: 945-953.

Reiland S, Messerli G, Baerenfaller K, Gerrits B, Endler A, Grossmann J, Gruissem W, Baginsky S (2009) Large-scale Arabidopsis phosphoproteome profiling reveals novel chloroplast kinase substrates and phosphorylation networks. Plant Physiol. 150, 889-903.

Roose JL, Wegener KM, Pakrasi HB (2007) The extrinsic proteins of Photosystem II. Photosynth. Res. 92, 369-387. 
Roose JL, Frankel LK, Bricker TM (2011) Developmental defects in mutants of the PsbP domain protein 5 in Arabidopsis thaliana. PLoS One. 6, e28624.

Sato N (2010) Phylogenomic and structural modeling analyses of the PsbP superfamily reveal multiple small segment additions in the evolution of photosystem II-associated PsbP protein in green plants. Mol. Phylogenet. Evol. 56, 176-86.

Schubert M, Petersson UA, Haas BJ, Funk C, Schröder WP, Kieselbach T (2002) Proteome map of the chloroplast lumen of Arabidopsis thaliana. J. Biol. Chem. 277, 8354-8365.

Schuhmann H, Adamska I (2012) Deg proteases and their role in protein quality control and processing in different subcellular compartments of the plant cell. Physiol. Plant. 145, 224-234.

Seidler A (1996) The extrinsic polypeptides of Photosystem II. Biochim. Biophys. Acta 1277, $35-60$.

Sirpiö S, Holmström M, Battchikova N, Aro EM (2009) AtCYP20-2 is an auxiliary protein of the chloroplast NAD(P)H dehydrogenase complex. FEBS Lett. 583, 2355-2358.

Sirpiö, S., Khrouchtchova, A., Allahverdiyeva, Y., Hansson, M., Fristedt, R., Vener, A.V. Scheller HV, Jensen PE, Haldrup A, Aro EM. (2008) AtCYP38 ensures early biogenesis, correct assembly and sustenance of Photosystem II. Plant J. 55, 639-51.

Sirpiö S, Allahverdiyeva Y, Suorsa M, Paakkarinen V, Vainonen J, Battchikova N, Aro EM. (2007) TLP18.3, a novel thylakoid lumen protein regulating photosystem II repair cycle. Biochem J. 406, 415-425.

Suorsa M, Sirpiö S, Aro EM (2009) Towards characterization of the chloroplast NAD(P)H dehydrogenase complex. Mol. Plant. 2, 1127-1140.

Suorsa M, Sirpiö S, Paakkarinen V, Kumari N, Holmström M, Aro EM (2010) Two proteins homologous to PsbQ are novel subunits of the chloroplast NAD(P)H dehydrogenase. Plant 
Cell Physiol. 51, 877-883.

Thornton LE, Ohkawa H, Roose JL, Kashino Y, Keren N, Pakrasi HB (2004) Homologs of plant PsbP and PsbQ proteins are necessary for regulation of photosystem II activity in the cyanobacterium Synechocystis 6803. Plant Cell, 16, 2164-7215.

Tomita M, Ifuku K, Sato F, Noguchi T (2009) FTIR evidence that the PsbP extrinsic protein induces protein conformational changes around the oxygen-evolving $\mathrm{Mn}$ cluster in photosystem II. Biochemistry 48, 6318-6325.

Ueda M, Kuniyoshi T, Yamamoto H, Sugimoto K, Ishizaki K, Kohchi T, Nishimura Y, Shikanai $\mathrm{T}$ (2012) Composition and physiological function of the chloroplast NADH dehydrogenase-like complex in Marchantia polymorpha. Plant J. 72, 683-693.

Uno C, Nagao R, Suzuki H, Tomo T, Noguchi T (2013) Structural coupling of extrinsic proteins with the oxygen-evolving center in red algal photosystem II as revealed by light-induced FTIR difference spectroscopy. Biochemistry. 52, 5705-5707.

Vasudevan D, Fu A, Luan S, Swaminathan K (2012) Crystal structure of Arabidopsis cyclophilin38 reveals a previously uncharacterized immunophilin fold and a possible autoinhibitory mechanism. Plant Cell 24, 2666-2674.

Wu HY, Liu MS, Lin TP, Cheng YS (2011) Structural and functional assays of AtTLP18.3 identify its novel acid phosphatase activity in thylakoid lumen. Plant Physiol. 157, 1015-1025.

Yabuta S, Ifuku K, Takabayashi A, Ishihara S, Ido K, Ishikawa N, Endo T, Sato F (2010) Three PsbQ-like proteins are required for the function of the chloroplast $\mathrm{NAD}(\mathrm{P}) \mathrm{H}$ dehydrogenase complex in Arabidopsis. Plant Cell Physiol. 51, 866-876.

Yamamoto H, Peng L, Fukao Y, Shikanai T (2011) An Src homology 3 domain-like fold protein forms a ferredoxin binding site for the chloroplast NADH dehydrogenase-like complex in Arabidopsis. Plant Cell. 23, 1480-1493. 
Yi X, Hargett SR, Frankel LK, Bricker TM (2006) The PsbQ protein is required in Arabidopsis for photosystem II assembly/stability and photoautotrophy under low light conditions (2006) J. Biol. Chem. 281, 26260-26267.

Yi X, Hargett SR, Liu H, Frankel LK, Bricker TM (2007) The PsbP protein is required for photosystem II complex assembly/stability and photoautotrophy in Arabidopsis thaliana. J. Biol. Chem. 282, 24833-24841.

Zybailov B, Rutschow H, Friso G, Rudella A, Emanuelsson O, Sun Q, van Wijk KJ (2008) Sorting signals, N-terminal modifications and abundance of the chloroplast proteome. PLoS ONE 4, e1994. 


\section{Figure legends}

Fig.1. Functional categories of thylakoid luminal proteins in chloroplasts. Only known and proposed functions of the lumen proteins experimentally identified by proteome studies are shown. Each protein name is described in the text or as in Järvi et al. (2013).

Fig.2. Schematic view of the relationship between PsbP and PsbQ binding, $\mathrm{Ca}^{2+}$ and $\mathrm{Cl}^{-}$ retention, and structural changes around Mn cluster. a) WT-PsbP has an N-terminal extension that interacts with PsbE in PSII. b) PsbP and PsbQ depletion triggers conformational change around the $\mathrm{Mn}$ cluster that weakens $\mathrm{Ca}^{2+}$ and $\mathrm{Cl}^{-}$retention capability. c) Depletion of PsbO does not cause further structural change. d) Reconstitution of PsbP alone can restore protein conformation of the OEC. e) N-terminal truncated $\Delta 15$-PsbP cannot restore protein conformation as well as $\mathrm{Ca}^{2+}$ and $\mathrm{Cl}^{-}$retention capability. f) PsbQ improves the binding of $\triangle 15-\mathrm{PsbP}$ and restores the OEC conformation and $\mathrm{Ca}^{2+}$ and $\mathrm{Cl}^{-}$retention in PSII.

Fig.3. Subcomplex organization of the chloroplast NDH-like complex in vascular plants. See the text for detail. 
Table 1. The PsbP and PsbQ family proteins in Arabidopsis

\begin{tabular}{|c|c|c|c|c|}
\hline Protein name & Other name & $\begin{array}{l}\text { Gene locus } \\
\text { (TAIR) }\end{array}$ & Proposed function & References \\
\hline PsbP1 & & At1g06680 & OEC & $\begin{array}{l}\text { Yi et al. 2007, } \\
\text { Allahverdiyeva et al. } \\
\text { 2013; }\end{array}$ \\
\hline PPL1 & & At3g55330 & PSII repair & Ishihara et al. 2007 \\
\hline PPL2 & PnsL1 & At2g39470 & NDH-like subunits & Ishihara et al. 2007 \\
\hline PPD1 & & At4g15510 & PSI assembly & Liu et al. 2012 \\
\hline PPD2 & & At2g28605 & $\begin{array}{l}\text { Singlet-oxygen } \\
\text { signaling } \\
\text { (Chlamydomonas) }\end{array}$ & $\begin{array}{l}\text { Brzezowski et al. } \\
2012\end{array}$ \\
\hline PPD3 & & At1g76450 & & Ifuku et al. 2010 \\
\hline PPD4 & & At1g77090 & & Ifuku et al. 2010 \\
\hline PPD5 & & At5g11450 & $\begin{array}{l}\text { Strigolactone } \\
\text { biosynthesis }\end{array}$ & Roose et al. 2011 \\
\hline PPD6 & & At3g56650 & & Ifuku et al. 2010 \\
\hline PPD7* & & At3g05410 & & Sato, 2010 \\
\hline PPD8* & & At5g27390 & & Järvi et al. 2013 \\
\hline PsbQ1 & & At4g21280 & OEC & $\begin{array}{l}\text { Yi et al. 2006; } \\
\text { Allahverdiyeva et al. } \\
2013\end{array}$ \\
\hline PsbQ2 & & At4g05180 & OEC & $\begin{array}{l}\text { Yi et al. 2006; } \\
\text { Allahverdiyeva et al. } \\
2013\end{array}$ \\
\hline PQL1/2 & PnsL2 & At1g14150 & NDH-like subunits & $\begin{array}{l}\text { Suorsa et al. 2010; } \\
\text { Yabuta et al. } 2010\end{array}$ \\
\hline PQL2/1 & PnsL3 & At3g01440 & NDH-like subunits & $\begin{array}{l}\text { Suorsa et al. 2010; } \\
\text { Yabuta et al. } 2010\end{array}$ \\
\hline PQL3* & & At2g01918 & NDH-like activity & Yabuta et al. 2010 \\
\hline
\end{tabular}




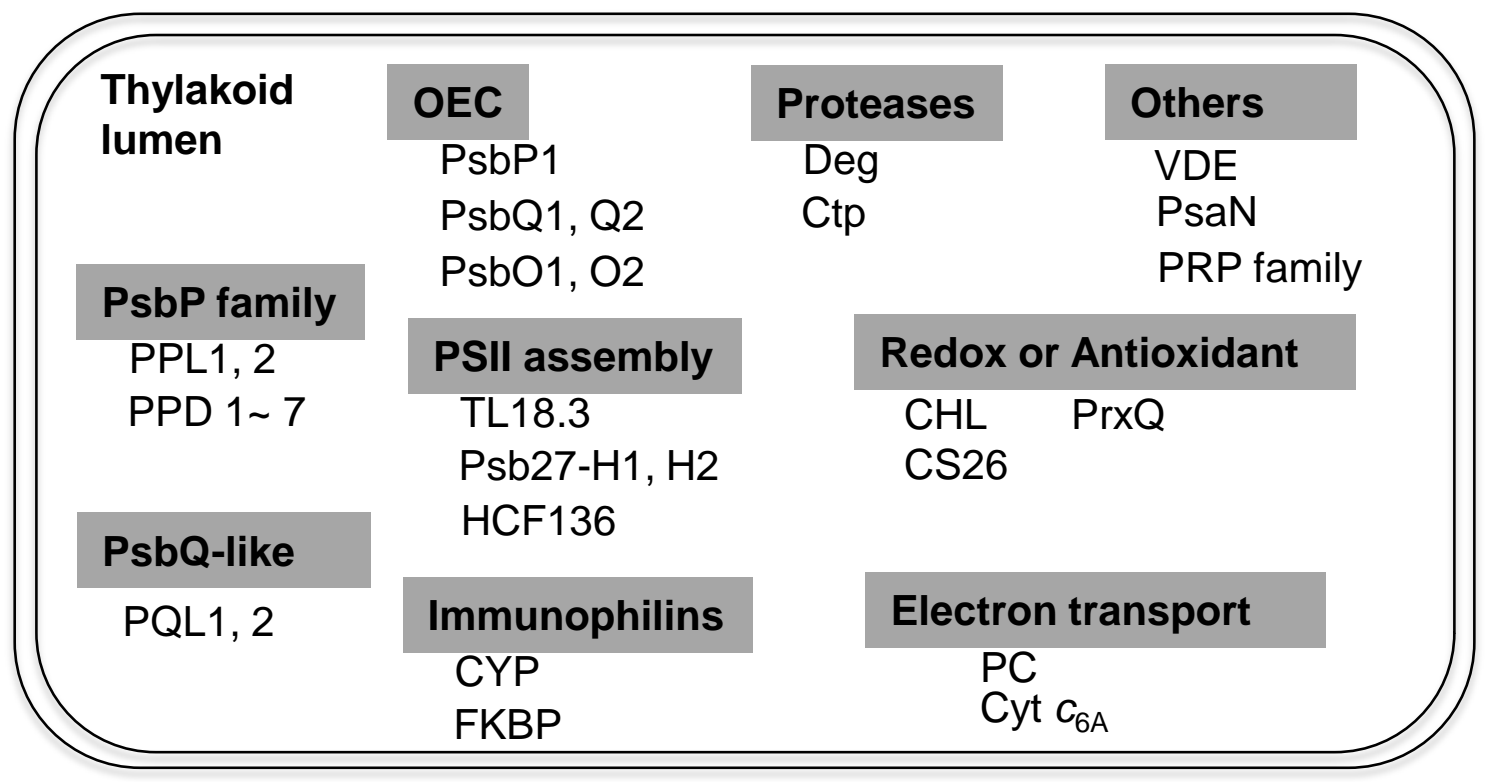

Fig. 1 
a)

d)
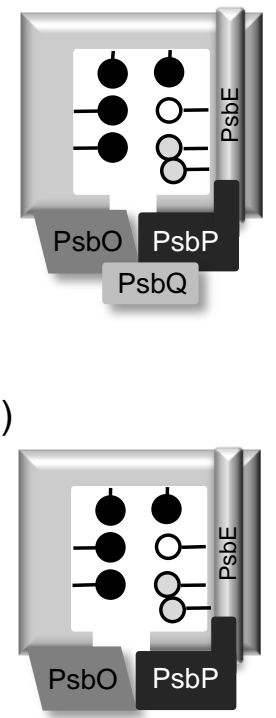

b)

$\downarrow$

e)

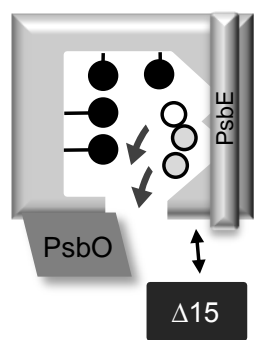

c)

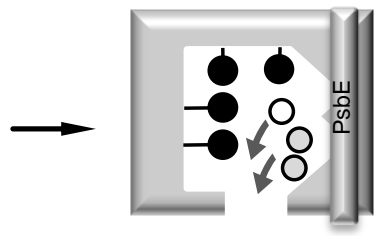

f)

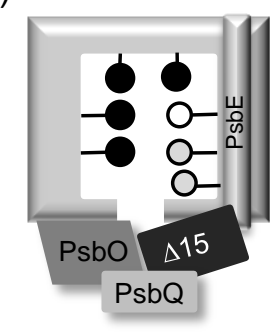

- $\mathrm{Mn} \mathrm{O} \mathrm{Ca}^{2+} \mathrm{O} \mathrm{Cl}^{-}$

Fig.2 


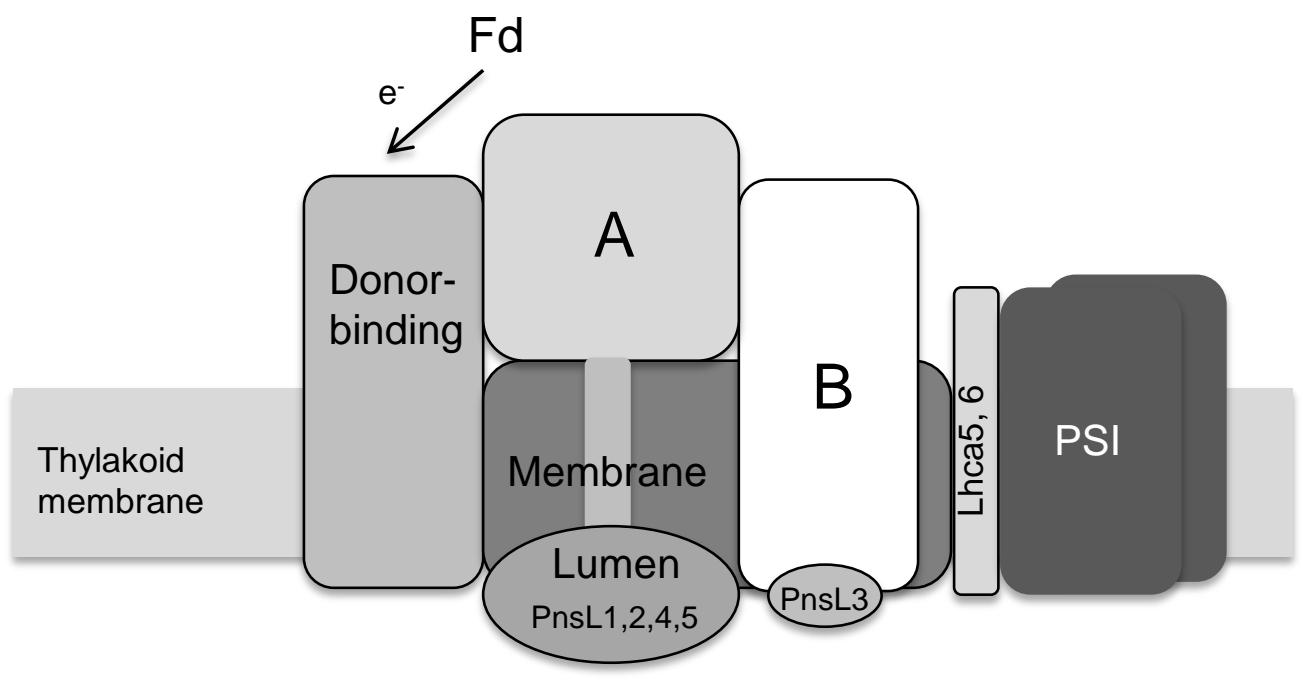

Fig.3 